\title{
Usability Evaluation of OpenWeb Transcoding
}

\author{
Elizabeth Uruchurtu, Ellie Lockley, Chris Roast \\ Communication and Computing Research Centre \\ Sheffield Hallam University \\ Arundel Street \\ Sheffield S1 2NU, UK \\ \{e.uruchurtu, e.lockley, c.r.roast\}@shu.ac.uk
}

\begin{abstract}
This paper describes collaborative work between industry and academia aimed at supporting the design and assessment of a transcoding service to support mobile phone internet browsing. The work demonstrates how research informed by user based assessment can be effectively and efficiently employed to inform and support industry. The paper describes a series of small scale, relatively rapid, evaluation studies that have focused on the comparative assessment of alternative transcoding approaches. These studies have: informed the design of OpenWeb transcoding; developed a number of easily efficient empirical assessment methods for mobile browsing; and provided a basis for focusing future user experience studies.
\end{abstract}

\section{Categories and Subject Descriptors}

H.5.2 [User Interfaces]: Evaluation/methodology, User-centered design. H.1.2 [User/Machine Systems]: Human factors. H.5.4 [Hypertext/Hypermedia] Navigation, User issues.

\section{General Terms}

Measurement, Design, Experimentation, Human Factors.

\section{Keywords}

Usability, Transcoding, Mobile Phone, Web Browsing.

\section{INTRODUCTION}

Transcoding is a technique that transforms content from the original format in which it was encoded into a second alternative format (Han \& Smith, 2001). Web content transcoding allows a wider collection of Internet-enabled client devices, such as mobile phones and hand-held devices, to have access to that content. The work reported in this paper focuses upon supporting browsing using standard mobile phones.

Openwave is one of the world's leading innovators of software applications and infrastructure for mobile and broadband operators. Openwave's product portfolio includes a range of mobile internet services, messaging and location based solutions. In particular, OpenWeb is a content adaptation platform that transparently intercepts requests for standard web sites and converts the web pages into highly compressed, functional WAP2 pages. Web pages are re-purposed for the mobile screen

Copyright is held by the author/owner(s).

MobileHCI'09, September 15 - 18, 2009, Bonn, Germany.

ACM 978-1-60558-281-8.

\author{
Inge De Bleecker \\ Openwave \\ Openwave Systems Inc. \\ 2100 Seaport Boulevard \\ Redwood City, CA 94063 USA \\ inge.debleecker@openwave.com
}

throughout a user session, on the fly.

The specific approach to OpenWeb transcoding of a web page can be outlined as follows:

1. DOM creation - A single-pass parse of the page source mark-up, building a full Document Object Model (DOM) and executing all "onLoad" Javascript scripts.

2. Form and event processing - The insertion of any form values the user has altered and execution of any Javascript events such as "onChange", "onSubmit", "onMouseOver", etc, that the user has triggered.

3. Build navigation and content - Run scripts to build navigation menu and identify the start of most relevant content.

4. Output - Output visible DOM contents to XHTML output buffer.

5. Split - Split the XHTML output buffer into sub-pages as required to make it work within memory of target phone.

6. Top and tail - Add the between sub-page navigation toolbars.

7. Send - Send to phone.

The Communication and Computing Research Centre (CCRC) at Sheffield Hallam University (SHU) has carried out a number of user evaluation studies on OpenWeb. The aim of these studies has been to gain a greater understanding of the importance of user perceptions and usability in determining attitudes to mobile phone Internet usage. The issues examined through these evaluations include user perceptions of ease of use, orientation, navigation and look and feel of the Web pages displayed on their mobile screens. These studies have been focused around representative tasks for mobile browsing. Specifically direct information seeking tasks have been assumed to be of most relevance for mobile browsing. User performance has then been assessed in terms of: efficiency the time taken to complete an information seeking task; and effectiveness the accuracy of the information found.

To be of most benefit to OpenWeb these studies have been comparative in nature. This has allowed alternative OpenWeb designs to be empirically compared, and also for alternative transcoding services to be compared with OpenWeb.

This paper describes and discusses the evaluation approach adopted and particularly how collaboration between SHU and Openwave demonstrates that research activity addressing industry concerns can be valuable to the parties involved. The paper concludes by describing how the studies have provided the basis for practical effective formative input into service design, and how this has helped direct future user experience studies.

\section{Evaluation studies}

A vast majority of publications of usability mobile browsing research focuses upon supporting browsing with: research 
applications such as bespoke mobile browser (de Bruijn and Spence 2000), or ambitious assumptions, such as the requirement for higher quality or specialised use of web page mark-up (Laakko \& Hiltunen, 2005). By contrast the usability value and impact of transcoding of websites as provided by OpenWeb has not been focused upon in detail. However, the challenge is to provide a proxy that does not have prescriptive control of either the client or server configuration/set-up, while still enabling and supporting effective browsing demands. Relatively recent developments have emphasised the importance of understanding how user experience is affected by transcoding and the resulting commercial impact (Wapreview 2009).

Accordingly, a genuine concern for Openwave has been to understand the extent to which their users understand and experience OpenWeb and other transcoding services.

An initial review of research regarding mobile usability highlighted a number of issues relevant for transcoding. Hyvärinen et al. (2005) for example, argue that there is overall preference for, and benefit to, navigation and services presented separately. Another important aspect refers to the models users develop from the websites they navigate. Evidence suggests that users tend to acquire a semantic model of a site as opposed to a physical model (Roto \& Kaikkonen 2003). These and other directly relevant research findings suggested that addressing Openwave concerns would require focusing on usability of the OpenWeb service in terms of ease of use, navigation structure and orientation.

Significant constraints for the studies were timescales and resources as individual OpenWeb design options were being developed, refined and selected. A user evaluation approach was therefore adopted based on a small scale study employing 'off the shelf' usability techniques to perform comparative evaluations. Limitations were also acknowledged in relation to past research which had demonstrated that usability measures taken in the field for mobile phones are significantly higher than those taken in laboratory/highly controlled settings (Duh et al. 2006). Therefore it was clear that usability issues identified by the evaluation study would likely to be more significant for users of the services in real practical contexts.

\section{Controlled Evaluation Design}

The first evaluation study design was based on a quiz answer view of mobile browsing in which subjects were given information seeking tasks. The study was conceptualised and promoted as a "pub quiz" in which participants were presented with a number of questions to be completed within a fixed period in a controlled setting. The participant with most correct answers would be rewarded with a small prize. Target subjects were characterised as mobile familiar and web familiar, and those not unfamiliar with accessing the Web via their mobile phone. In turn, after experiencing the service and completing the quiz, measures were taken in terms of subjective ease of use to assess usability and orientation (see Ahuja, 2001); subjects' perceptions of, and reactions to, the service.

The participants were higher education students aged between 18 and 39 years (average: 22 years), the majority of subjects were undergraduates.

\subsection{The initial study}

Using the controlled design, the initial study compared OpenWeb design alternatives with two specific transcoding configurations which included different approaches to blocking pages. This was performed as between-group study using the "pub quiz" model.

Tasks were arranged as simple factual questions set for three types of websites: one that users were likely to be familiar with (in this case Amazon.com); one judged to unfamiliar to them (the "New Brunswick" council site); and one with rich functionality (the UK National Rail site).

Results from this initial evaluation indicated that there was a general agreement among participants that OpenWeb was an efficient way of accessing the Web via mobile devices and that it was easy to learn and to use. There were not significant differences in terms of user performance between the configuration options examined. Differences in terms of orientation related to the amount of information displayed at a time between the different configurations used: breaking the content into expandable blocks was perceived as more navigable than the non-blocking approach. Blocking coincided with background research (Hyvärinen et al. 2005) which showed that it is important to make navigation links within a site distinct to service links. Since the benefits of this separation are reliant upon the users understanding of how a transcoded site may appear different, further enhancements of the service were identified in the possibility of avoiding this separation by making the transcoding service completely transparent for its users.

This initial study was of value in validating the approach taken and providing relatively rapid feedback regarding the two alternative configurations. This information could be incorporated back into the design and refinement of the OpenWeb content adaptation engine.

\subsection{Repeated Studies}

Three more evaluation studies have been carried out to date on the OpenWeb transcoding service using the "pub quiz" model. In line with the initial aim of the collaboration between SHU and Openwave, these studies have examined the effectiveness, efficiency, usability and user orientation of OpenWeb. The specific approach, tasks and questionnaire have remained largely the same to enable cross study comparisons.

The specific comparative focus of the studies was to examine different configuration options and to compare the use of OpenWeb with other similar services.

Findings resulting from these studies as a whole show that although transcoding is primarily driven by analytic and technical performance, user experience and perception can be at odds with more objective measures. General findings arising from these studies have shown that:

- The subjective measures taken relating usability and navigability have concurred. Thus, when two alternative transcodings are compared, the more usable is also the more navigable.

- When comparing two transcoding alternatives, the alternative receiving the higher usability rating has also been the slowest for task completion.

- Task efficiency and effectiveness do not appear to be consistently related.

- The post questionnaire used to assess subjective views of navigability and usability, consistently for all transcodings produced results where navigability is rated lower than usability. 
Findings from these studies have been employed to help direct and inform future work and also been used constructively in the development of OpenWeb transcoding. In particular, a number of alternative algorithms for the transcoding steps 3 Build navigation and content and 5 Split (see above) have been tried, and their selection has been supported by the results from these user facing studies.

\subsection{Physically Engaged Evaluation Study Design}

Although the "pub quiz" model of evaluation has been of value, research on mobile usage shows that it may be missing important features of mobile usability. As a result the most recent study (December 2008) was re-designed to be more physically engaging for the participants. The reason for modifying the experimental design was to take account of the tendency for static usability assessment to over emphasise cosmetic usability issues.

A 'physically mobile' evaluation approach was developed to address this point and also to provide a more interesting user experience. The new approach gathered the same pre and post study information and feedback, but in addition provided quiz questions in a randomised sequence for each subject. The questions were also presented on several sheets which were distributed through one floor of the Faculty building. This meant that the subjects were forced to be physically mobile since they were walking while browsing.

The disadvantage of having subjects free to move around an open area is the loss of control. Subjects would in principle be free to exchange answers to questions and also access the internet through Faculty workstations. To limit the first of these possibilities, a broad range of potential questions were used. Hence, subjects could not predict if each other were in fact answering the same question. In addition to ensure the use of their mobile handsets for answering the questions, subjects were required to report back their answers to the research team by showing the relevant webpage on their mobile phone screen.

Content adaptation alternatives have been randomly assigned in all cases and tasks arranged as factual questions for familiar and unfamiliar websites. Mobile phone models have not been a controlled variable.

The results from this new form of study have been positive in two respects. First as planned this new form of study generated more positive responses to transcoding and web browsing and engaged subjects. Subjects were clearly less concerned with taking part when compared with the more controlled setting of earlier studies. In line with our expectations for this new form of study, both usability and navigability were rated far higher. Second, the results from this study have been sufficiently interesting to initiate the design of another further study currently being planned.

\section{Results}

In general, mobile access to the Web is perceived as positive and convenient. Differences in performance have been observed during the evaluation studies however they are not statistically significant. Similarly, differences in user performance between OpenWeb and other services have been observed, but were not found to be significant. Participants' perceptions suggest that websites are expected to look the same as their PC experience; therefore, differences in layout, look and feel and amount of text or images can affect the way usability and navigability is judged. Performance of the transcoding service has also been highlighted as an influential factor, in particular loading and processing times. In general, it appears that users are familiar with their mobile phones resources and capabilities and work effectively using them. Thus, users are capable of accommodating the features of their client mobile and the service provider.

In addition to the above points it can be seen that subject perceptions of mobile based browsing are developing. In particular, looking across studies from mid-2007 to mid-2008 a general trend has been for mobile browsing to be more common. One potentially related factor is that with time fewer subjects have expressed concern with the likely expense of using their mobile phone to browse the internet.

\subsection{Conclusions and Future Work}

In describing collaborative work between industry and academia this paper has shown that research informed by user based assessment can be effective and efficient in informing design and supporting industry. Often the demands and interests of these two parties are at odds. Despite this the development of relative rapid and resource light evaluation methods has enabled more effective collaboration. In effect, the process of collaboration has been incremental, over time evaluation approaches have improved and focused upon specific industry needs.

The most recent research in combination with the earlier findings has led to the design of a future study. This new study will focus upon achieving a more accurate understanding of how subjects understand transcoded content. For instance, whether they "see the transcoding" or "see through the transcoding". The study is planned to be more qualitative in nature and will gather specific measures to allow for comparisons with the previous studies.

Other areas of future collaborative research and evaluation which could be of specific benefit include:

- Examining the value of making more configuration options "user configurable". In particular, users will understand the options and subsequently learn about their impact when browsing. It would be worth exploring alternative transcoding strategies based upon the integration pre-analysis of site use and appropriate dynamic user configuration options.

- As the accepted use and uses of the internet develop, the value of mobile browsing is likely to focus upon access to more advanced, and functionally rich, internet applications. One area of particular interest involves mobile access to social networking. Studies with such a focus are likely to require longitudinal data gathering and the identification of social factors relevant to, and influenced by, effective social networking.

- Methodologically it would be of particular value to further develop efficient and robust approaches to conducting experienced and focused assessments. In particular, developing methods that can be employed to effectively meet the needs of industrial partners.

\section{ACKNOWLEDGMENTS}

Our thanks to Robert Finean and Ed Moore of Openwave, and Openwave's development team for helping to manage the studies described above. Our thanks also go to our colleague Terry Keefe 
who assisted with the early studies and to the students who participated in the studies.

\section{REFERENCES}

[1] Ahuja, J.S. \& Webster, J. (2001) Perceived disorientation: an examination of a new measure to assess Web design effectiveness. Interacting with computers Vol. 14, pp. 15-29.

[2] De Bruijn, O. and Spence, R. (2000). Rapid serial visual presentation: a space-time trade-off in information presentation. In Proceedings of the Working Conference on Advanced Visual interfaces (Palermo, Italy). AVI '00. ACM, New York, NY, 189-192. DOI= http://doi.acm.org/10.1145/345513.345309

[3] Duh, H.B-L, Tan, G.C.B. \& Chen, V.H-H (2006) Usability evaluation for mobile device: a comparison of laboratory and field tests. Proceedings of the 8th ACM Conference on Human-Computer Interaction with mobile devices and services, pp. 181 - 186. http://doi.acm.org/10.1145/1152215.1152254

[4] Han R. \& Smith J. R. (2001) Transcoding of the Internet's multimedia Content for Universal Access. In Gibson J. D Multimedia communications. San Diego: Academic Press.
[5] Hyvärinen, T., Kaikkonen, A. \& Hiltunen, M. (2005) Placing links in mobile banking application. Proceedings of the 7th ACM International Conference on Human-Computer Interaction with mobile devices \& services. Salzburg, Austria, pp. 63 - 68 http://doi.acm.org/10.1145/1085777.1085788

[6] Laakko, T. \& Hiltunen, T. (2005) Adapting Web content to mobile user agents. Internet Computing, IEEE Vol. 9(2), pp. 46-53

[7] Oulasvirta A. et al. (2005) Interaction in 4-second burst: the fragmented nature of attentional resources in mobile HCI. Proceeding of the SIGCHI conference on Human Factors in computing systems, pp. $919-928$.

[8] Roto V. \& Kaikkonen A. (2003) Perception of narrow web pages on a mobile phone. Proceedings of the 19th International Symposium on Human Factors in Telecommunications. Berlin, Germany, December 1-4, 2003.

[9] Wapreview - "Opting out of Transcoding", http://wapreview.com/blog/?p=918, accessed Feb 2009. 